\title{
Synthesis, Crystal Structure, Catalytic Properties, and Luminescent of a Novel Eu(III) Complex Material with 4-Imidazolecarboxaldehyde-pyridine-2-carbohydrazone
}

\author{
Li-Hua Wang1,"*, Lei Liang'2, Peng-Fei Li ${ }^{3}$ \\ ${ }^{1}$ College of Information and Engineering, Weifang University, Weifang 261061, P.R. China \\ ${ }^{2}$ School of Harbin Light Industry, Harbin 150040, P.R. China \\ ${ }^{3}$ College of Chemical Engineering, Qingdao University of Science and Technology, \\ Qingdao 266061, P.R. China
}

Received: 11 ${ }^{\text {st }}$ November 2016; Revised: $10^{\text {th }}$ February 2017; Accepted: $23^{\text {rd }}$ February 2017

\section{Abstract}

A novel Eu(III) complex, $\left[\mathrm{Eu}(\mathrm{L})_{2}\left(\mathrm{H}_{2} \mathrm{O}\right)_{4}\right] \cdot\left(\mathrm{NO}_{3}\right) \cdot\left(\mathrm{H}_{2} \mathrm{O}\right)_{4}(\mathbf{1})\left(\mathrm{H}_{2} \mathrm{~L}=4\right.$-imidazolecarboxaldehyde-pyridine-2carbohydrazide), was synthesized. Its structure has been characterized by elemental analysis, IR, and X-ray single crystal diffraction analysis. Complex 1 is of orthorhombic, space group Fdd2 with $a=$ 29.471(6) $\mathrm{A}^{\circ}, b=10.287(2) \mathrm{A}^{\circ}, c=24.340(5) \mathrm{A}^{\circ}, V=7379(3) \mathrm{A}^{\circ} 3, Z=8, M r=902.58, D c=1.625 \mu \mathrm{g} \cdot \mathrm{m}^{-3}$, $\mu=1.789 \mathrm{~mm}^{-1}, F(000)=3656, G O O F=1.099$, the final $R=0.0517, \omega R=0.1292$ for 3043 observed reflections with $I>2 \sigma(\mathrm{I})$. The $\mathrm{A}^{3}$ coupling reaction has been investigated using the complex 1 as catalyst. The luminescent spectrum of the complex 1 gives two weak peaks (448 nm and $491 \mathrm{~nm}$ ) and two strong peaks (596 nm and $620 \mathrm{~nm}$ ) from excitation at $279 \mathrm{~nm}$. Copyright () 2017 BCREC Group. All rights reserved.

Keywords: $\mathrm{Eu}(\mathrm{III})$ complex; Luminescence; pyridine; carbohydrazone; Catalyst

How to Cite: Wang, L.H., Liang, L., Li, P.F. (2017). Synthesis, Crystal Structure, Catalytic Properties, and Luminescent of a Novel Eu(III) Complex Material with 4-Imidazolecarboxaldehyde-pyridine-2carbohydrazone. Bulletin of Chemical Reaction Engineering \& Catalysis, 12 (2): 185-190 (doi:10.9767/bcrec.12.2.764.185-190)

Permalink/DOI: http://dx.doi.org/10.9767/bcrec.12.2.764.185-190

\section{Introduction}

Increasing studies have been focused on the europium complexes, because they show rich coordination model, biological activity, catalytic activity and strong red luminescence [1-4]. So far, various $\mathrm{Eu}(\mathrm{III})$ complexes with multipodal ligands, such as diketonate [5], Schiff-base [6], carboxylate [7], and bipyridyl [8] have been reported. Meanwhile, hydrazone compounds are excellent multipodal ligands [9], and their me-

* Corresponding Author.

E-mail: wanglihua929@163.com (Wang, L.H.)

Telp.: +86-536-8785613; Fax.: +86-536-8785613 tal complexes have also shown good properties in cytotoxic activity [10], luminescence [11], and catalytic application [12]. Syntheses of propargylamines under heterogeneous catalysis are particularly attractive as they allow the production and ready separation of large quantities of products using small amounts of catalysts. So the development of heterogeneous catalysts for preparing propargylamines by the $\mathrm{A}^{3}$ coupling reaction remains an active research area [1317]. However, so far, there is little research on the catalytic activity of $\mathrm{Eu}(\mathrm{III})$ complexes. As part of our research interest, in this paper, a novel $\mathrm{Eu}(\mathrm{III})$ complex was reported. Moreover, 
the synthesis, crystal structure, catalytic activity and luminescent property of $\mathrm{Eu}(\mathrm{III})$ complex have also been investigated. The scheme of 4 imidazolecarboxaldehyde-pyridine-2 carbohydrazide is given in Figure 1.

\section{Materials and Method}

\subsection{Materials and measurements}

4-Imidazolecarboxaldehyde (A. R.), pyridine2-carbohydrazide (A.R.) and $\mathrm{Eu}\left(\mathrm{NO}_{3}\right)_{3} \cdot 6 \mathrm{H}_{2} \mathrm{O}$ (A.R.) were purchased from Aladdin Reagent Company. The analyses of $\mathrm{C}, \mathrm{H}$, and $\mathrm{N}$ were made on a Elementar Vario EL III elemental analyzer. Infrared spectra ( $\mathrm{KBr}$ pellets, 4000$400 \mathrm{~cm}^{-1}$ ) were recorded on a Nicolet NEXUS 670 spectrophotometer. The X-ray singlecrystal data collection for complex 1 was per-

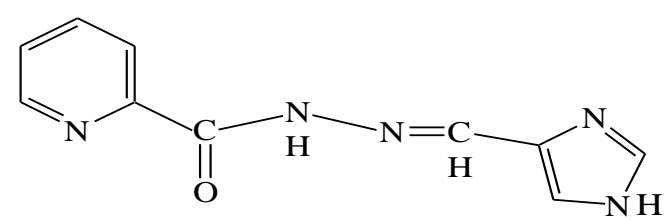

Figure 1. The scheme of $\mathrm{H}_{2} \mathrm{~L}$ formed on a Bruker Smart-1000 CCD diffractometer. The luminescent emission spectrum of complex $\mathbf{1}$ in solid state was measured on a PE LS-55 spectrometer.

\subsection{Synthesis of 4-imidazolecarboxaldehy- de-pyridine-2-carbohydrazide}

A mixture of 4-imidazolecarboxaldehyde $(0.9609 \mathrm{~g}, \quad 1.0 \mathrm{mmol})$ and pyridine-2carbohydrazide $(0.1371 \mathrm{~g}, 1.0 \mathrm{mmol})$ in $5 \mathrm{~mL}$ $\mathrm{CH}_{3} \mathrm{CH}_{2} \mathrm{OH}$ was refluxed for $6 \mathrm{~h}$. The precipitate of 4-imidazolecarboxaldehyde-pyridine-2carbohydrazide was filtered off and dried. Elemental analysis calc. for $\mathrm{C}_{10} \mathrm{H}_{9} \mathrm{~N}_{5} \mathrm{O}: \mathrm{C}, 55.81$, H, 4.19, N, 32.56 (\%); Found: C, 55.62, H, 3.86, $\mathrm{N}, 32.83(\%)$. IR: $3356 \mathrm{~cm}^{-1}\left(\mathrm{H}_{2} \mathrm{O}\right), 1662 \mathrm{~cm}^{-1}$ $(\mathrm{C}=\mathrm{O}), 1652 \mathrm{~cm}^{-1}(\mathrm{C}=\mathrm{N})$.

\subsection{Synthesis of complex 1}

The 4-Imidazolecarboxaldehyde-pyridine-2carbohydrazide $(0.430 \mathrm{~g}, 2.0 \mathrm{mmol})$ and $\mathrm{Eu}\left(\mathrm{NO}_{3}\right)_{3} \cdot 6 \mathrm{H}_{2} \mathrm{O}(0.4461 \mathrm{~g}, 1.0 \mathrm{mmol})$ were dissolved in $10 \mathrm{~mL} \mathrm{H} \mathrm{H}_{2} \mathrm{O} / \mathrm{CH}_{3} \mathrm{CH}_{2} \mathrm{OH}$ (v:v=1:1). The solution mixture was heated at $50{ }^{\circ} \mathrm{C}$ for $6 \mathrm{~h}$. The resultant solution was filtered and left

Table 1. The most crystal data of complex 1

\begin{tabular}{|c|c|}
\hline Empirical formula & $\mathrm{C}_{20} \mathrm{H}_{32} \mathrm{~N}_{11} \mathrm{O}_{13} \mathrm{Eu}$ \\
\hline Formula weight & 786.50 \\
\hline Temperature/K & 293(2) \\
\hline Crystal system & orthorhombic \\
\hline Space group & FDD2 \\
\hline $\mathrm{a} / \AA$ & $29.471(6)$ \\
\hline $\mathrm{b} / \AA$ & $10.287(2)$ \\
\hline$c / \AA$ & $24.340(5)$ \\
\hline$a=\beta=\gamma /^{\circ}$ & 90.00 \\
\hline Volume/Å3 & $7379(3)$ \\
\hline $\mathrm{Z}$ & 8 \\
\hline$\rho_{\text {calc }} \mathrm{mg} / \mathrm{mm}^{3}$ & 1.625 \\
\hline$\mu / \mathrm{mm}^{-1}$ & 1.789 \\
\hline$S$ & 1.115 \\
\hline$F(000)$ & 3656 \\
\hline Index ranges & $\begin{array}{l}-34 \leq h \leq 34 \\
-11 \leq k \leq 11 \\
-28 \leq l \leq 28\end{array}$ \\
\hline Reflections collected & 13511 \\
\hline Independent reflections & $3214[\mathrm{R}(\mathrm{int})=0.071]$ \\
\hline Data/restraints/parameters & $3214 / 6 / 236$ \\
\hline Goodness-of-fit on F2 & 1.082 \\
\hline$R_{1}, w R_{2}$ [all data] & $0.0531,0.1306$ \\
\hline$R_{1}, w R_{2}[I>2 \sigma(I)]$ & $0.0517,0.1292$ \\
\hline
\end{tabular}


aside for crystallization at room temperature. The crystals appeared for 30 days which were collected by filtration. Elemental analysis calc. for $\mathrm{C}_{20} \mathrm{H}_{32} \mathrm{~N}_{11} \mathrm{O}_{13} \mathrm{Eu}$ : C, 30.51, H, 4.07, N, 19.58 (\%); Found: C, 30.32, H, 4.36, N, 19.81 (\%). IR: $1622 \mathrm{~cm}^{-1}(\mathrm{C}=\mathrm{O}), 1619 \mathrm{~cm}^{-1}(\mathrm{C}=\mathrm{N})$.

\subsection{Crystal data and structure determina- tion}

X-ray diffraction analysis of complex 1 was carried out at 293 (2) K on a Bruker Smart1000 CCD diffractometer by using a $\varphi \sim \omega$ scan mode. The structure was solved by direct methods with SHELXL-97 program package [18] and refined with SHELXTL-97 [19]. The most crystal data of complex 1 are listed in Table 1.

\subsection{General procedure for the three com- ponent coupling reaction $\left(\mathrm{A}^{3}\right)$}

The coupling of benzaldehyde, piperidine and phenylacetylene with 1,4-dioxane as solvent was selected as a model reaction. The reaction was conducted by heating Eu(III) complex catalyst $(70 \mathrm{mg})$, benzaldehyde $(0.13$ $\mathrm{mmol})$, piperidine $(0.15 \mathrm{mmol})$, and phenylacetylene $(0.17 \mathrm{mmol})$ in 1,4 -dioxane $(1.5 \mathrm{~g})$ without any additives or an inert atmosphere at 120 ${ }^{\circ} \mathrm{C}$ for $5 \mathrm{~h}$. The yield of propargylamine is equal to the conversion of benzaldehyde, and was determined by using a by GC (GC1100, Capillary Column, SE-54) analysis.

\section{Results and Discussion}

\subsection{Structural description}

The complex 1 crystallizes in orthorhombic space group FDD2. The crystal structure of complex $\mathbf{1}$ is constructed by a central Eu(III) ion, two 4-imidazolecarboxaldehyde-pyridine-2carbohydrazide anions, a isolated $\mathrm{NO}_{3}$ - counter anion, four coordinated water molecules and four lattice water molecules (Figure 2). The $\mathrm{Eu}(\mathrm{III})$ ion is ten-coordinated with two oxygen atoms ( 1 and O1A) of 4imidazolecarboxaldehyde-pyridine-2carbohydrazide ligand, four nitrogen atoms (N1, N1A, N3 and N3A) of 4imidazolecarboxaldehyde - pyridine - 2 carbohydrazide ligand, and four oxygen atoms (O1W, O1WA, O2W and O2WA) of coordinated water molecules.

There have four five-membered rings (ring 1: Eu1-O1-C5-N4-N3-Eu1, ring 2: Eu1-N1-C3C4-N3-Eu1, ring3: Eu1-O1A-C5A-N4A-N3AEu1, ring 4: Eu1-N1A-C3A-C4A-N3A-Eu1) around central Eu(III) ion. The dihedral angles are $0.7^{\circ}$ (ring 1 and ring 2 ), $4.2^{\circ}$ (pyridine ring and imidazole ring), $1.6^{\circ}$ (ring 1 and imidazole ring), $3.3^{\circ}$ (ring 1 and pyridine ring), 3.9 (ring 2 and pyridine ring), $1.0^{\circ}$ (ring 2 and imidazole ring), respectively. Which show that the molecule is almost coplanar. The bond lengths of $\mathrm{Eu}-\mathrm{O}$ and $\mathrm{Eu}-\mathrm{N}$ are accordance with those of reported in the literature [20].
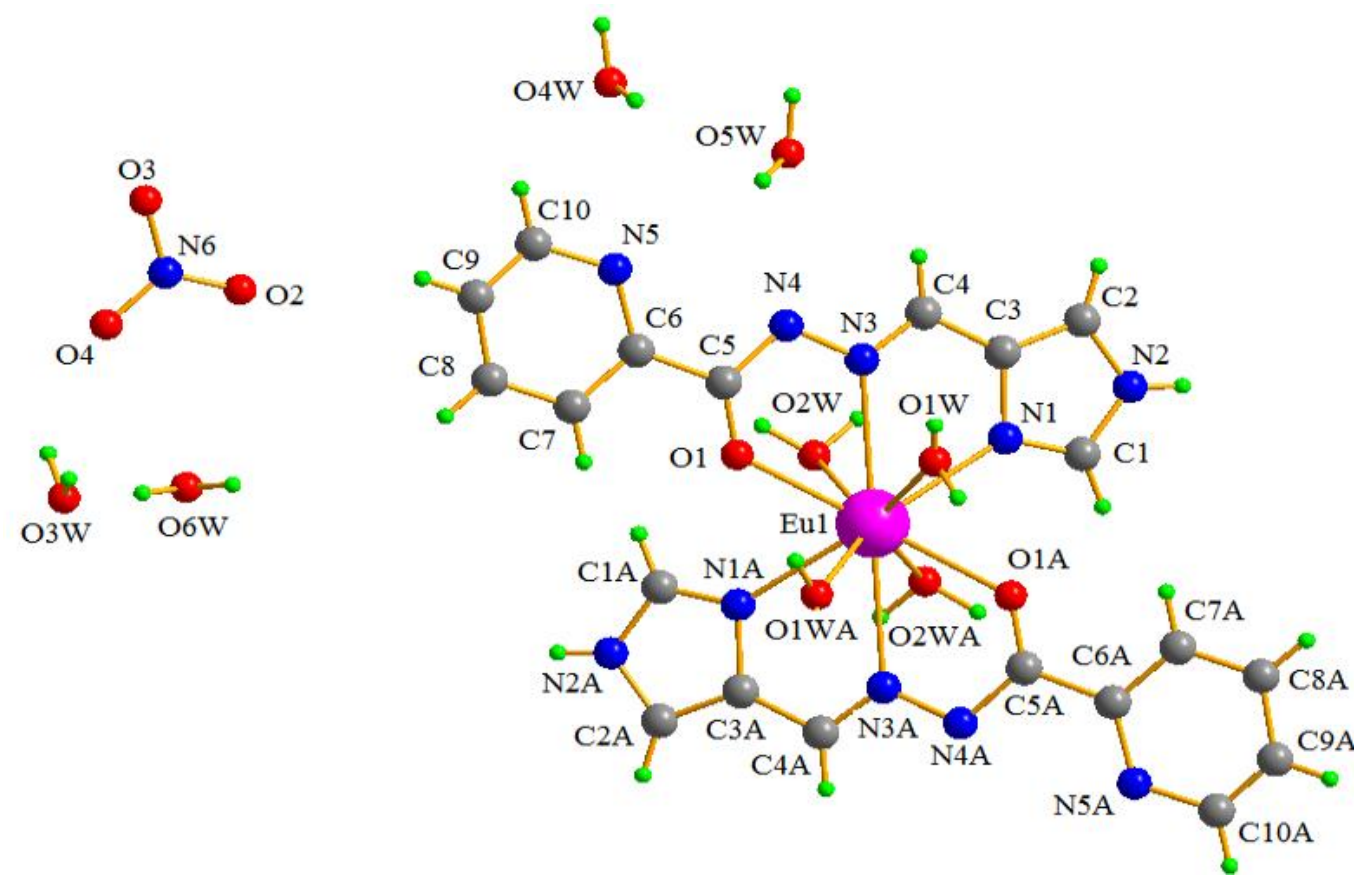

Figure 2. Molecular structure of complex 1, symmetry code: $-x+3 / 2,-y-3 / 2, z$ 
The complex molecules form 3D network structure by the aid of intermolecular hydrogen bonds and intramolecular hydrogen bonds (Table 2 and Figure 3).

Bonds for 1: Eu1-O2WA 2.479(6) ^; Eu1-O2W

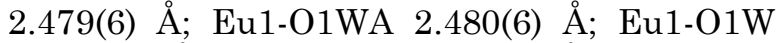

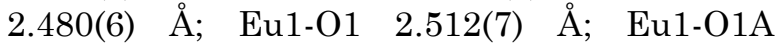

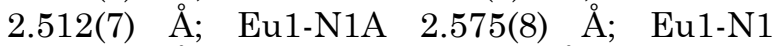

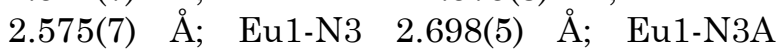

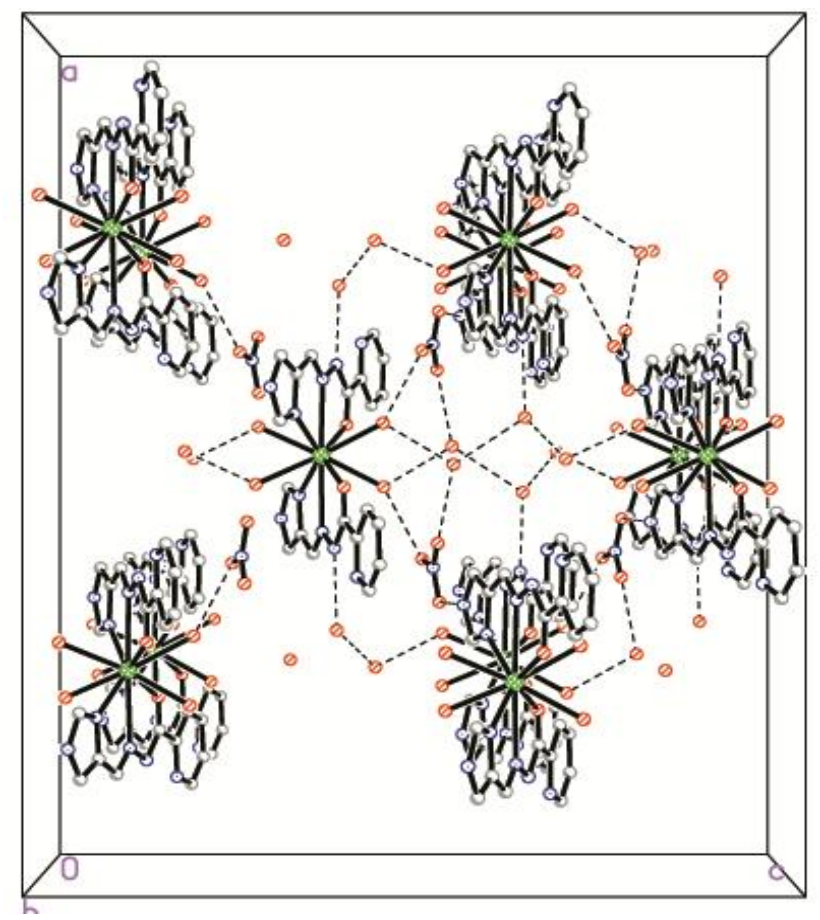

Figure 3. Packing of complex 1

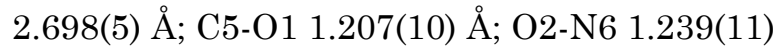

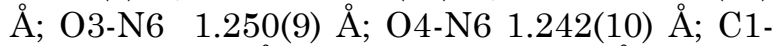

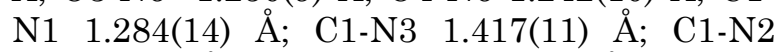
1.346(12) $\AA$; $\quad \mathrm{N} 2-\mathrm{C} 2 \quad 1.356(12) \quad \AA ; \quad \mathrm{C} 4-\mathrm{N} 3$ 1.255(10) $\AA$; $\quad \mathrm{N} 3-\mathrm{N} 4 \quad 1.365(9) \quad \AA ; \quad \mathrm{C} 5-\mathrm{N} 4$ 1.374(10) Å; N5-C6 1.372(12) Å; N5-C10 1.374(11) A; $\quad$ C2-C3 1.394(12) A; C3-C4 1.452(11) $\mathrm{A} ; \quad \mathrm{C} 5-\mathrm{C} 6 \quad 1.507(10) \quad \AA ; \quad \mathrm{C} 6-\mathrm{C} 7$ 1.383(12) Å; C7-C8 1.387(14) Å; C8-C9

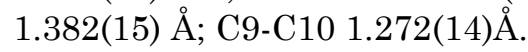

Angles for 1: O2W-Eu1-O2WA 65.1(3) ${ }^{\circ}$; O2WA-Eu1-O1WA 129.14(16) ${ }^{\circ}$; O2W-Eu1O1WA $138.83(16)^{\circ}$; O $2 \mathrm{WA}-\mathrm{Eu} 1-\mathrm{O} 1 \mathrm{~W}$ 138.83(16) ${ }^{\circ}$; O2W-Eu1-O1W 129.14(16) ${ }^{\circ}$; O1WEu1-O1WA 69.6(3) ${ }^{\circ}$; O2WA-Eu1-O1 137.7(2) ${ }^{\circ}$; O2W-Eu1-O1 77.9(2) ${ }^{\circ}$; O1-Eu1-O1WA 69.2(2) ${ }^{\circ}$; O1-Eu1-O1W 80.7(2) ${ }^{\circ}$ O O2WA-Eu1-O1A $77.9(2)^{\circ}$; O2W-Eu1-O1A 137.7(2) ${ }^{\circ}$; O1A-Eu1O1WA 80.7(2) ${ }^{\circ}$; O1A-Eu1-O1W 69.2(2) ${ }^{\circ}$; O1AEu1-O1 143.3(3) ${ }^{\circ}$; N1A-Eu1-O2WA 77.7(2) ${ }^{\circ}$; N1A-Eu1-O2W 72.1(2) ${ }^{\circ}$; N1A-Eu1-O1WA 74.5(2) ${ }^{\circ}$; N1A-Eu1-O1W 140.7(2) ; N1A-Eu1O1 71.8(2) ${ }^{\circ}$; N1A-Eu1-O1A $120.4(2)^{\circ}$; N1-Eu1O2WA 72.1(2) ${ }^{\circ}$; N1-Eu1-O2W 77.7(2) ${ }^{\circ}$; N1Eu1-O1WA 140.7(2) ${ }^{\circ}$; N1-Eu1-O1W 74.5(2) ${ }^{\circ}$; N1-Eu1-O1 120.5(2) ${ }^{\circ}$; N1-Eu1-O1A 71.8(2) ${ }^{\circ}$; N1-Eu1-N1A 144.1(4) ${ }^{\circ}$; N3-Eu1-O2WA $116.9(3)^{\circ} ; \quad \mathrm{N} 3-\mathrm{Eu} 1-\mathrm{O} 2 \mathrm{~W} \quad 65.0(3)^{\circ}$; N3-Eu1O1WA 113.8(3) ${ }^{\circ}$; N3-Eu1-O1W 64.4(3) ${ }^{\circ}$; N3Eu1-O1 58.91(19) ${ }^{\circ}$; N3-Eu1-O1A 120.4(2) ${ }^{\circ}$; N3Eu1-N1A 119.2(2) ${ }^{\circ}$; N3-Eu1-N1 61.5(2) ${ }^{\circ}$; N3AEu1-O2WA 65.0(3) ${ }^{\circ}$; N3A-Eu1-O2W 116.9(3) ${ }^{\circ}$; N3A-Eu1-O1WA $64.4(3)^{\circ} ; \quad$ N3A-Eu1-O1W

Table 2. Hydrogen bonds for complex 1

\begin{tabular}{lllll}
\hline D-H...A & $d(\mathrm{D}-\mathrm{H})$ & $d(\mathrm{H} \ldots \mathrm{A})$ & $d(\mathrm{D} \ldots \mathrm{A})$ & $\angle \mathrm{DHA}$ \\
\hline O1W-H1WB...O2 & 0.66 & 2.22 & $2.871(10)$ & 171 \\
O1W-H1WA...O4W & 0.87 & 1.97 & $2.795(12)$ & 159 \\
N2-H2B...O3 & 0.86 & 2.04 & $2.896(10)$ & 171 \\
N2-H2B...O4 & 0.86 & 2.51 & $3.136(10)$ & 130 \\
O2W-H2WB...O2 & 0.96 & 2.43 & $3.044(10)$ & 122 \\
O2W-H2WB...O3 & 0.96 & 2.10 & $3.013(9)$ & 159 \\
O3W-H3WD...O2W & 0.96 & 1.85 & $2.711(11)$ & 148 \\
O3W-H3WB...O5W & 0.96 & 2.11 & $2.849(12)$ & 132 \\
O4W-H4WA...O5W & 0.86 & 2.48 & $2.890(13)$ & 110 \\
O4W-H4WB...O5W & 0.96 & 2.47 & $2.890(13)$ & 106 \\
O5W-H5WA...O3W & 0.96 & 2.41 & $2.849(12)$ & 107 \\
O6W-H6WD...O3W & 0.96 & 2.54 & $3.466(17)$ & 163 \\
\hline
\end{tabular}


113.8(3) ${ }^{\circ}$; N3A-Eu1-O1 120.4(2) ${ }^{\circ}$; N3A-Eu1O1A 58.91(19) ${ }^{\circ}$; N3A-Eu1-N1A 61.5(2) ${ }^{\circ}$; N3AEu1-N1 119.2(2) ${ }^{\circ}$; N3A-Eu1-N3 178.0(6) ${ }^{\circ}$.

\subsection{Catalytic studies}

The prepared complex was used as a catalyst for $\mathrm{A}^{3}$ coupling reaction of aldehyde, amine and terminal alkyne (Figure 4). The yield of propargylamine was determined by using GC (GC1100, Capillary Column, SE-54) analysis. The results exhibit that only a trace amount of propargylamine was obtained when do not use any catalyst. A yield of $2.8 \%$ was obtained at $120{ }^{\circ} \mathrm{C}$ for $5 \mathrm{~h}$. The yield of propargylamine was reached $16 \%$ for $5 \mathrm{~h}$ at $120{ }^{\circ} \mathrm{C}$ using complex 1 as a catalyst. It can be seen that the complex has a higher yield of propargylamine than blank. However, the activity of complex is still less than those of $\mathrm{Au} / \mathrm{MOF}$ and $\mathrm{Cu}(\mathrm{II})$ complex material $[6,15]$.

\subsection{Luminescent property}

The luminescent properties of Eu(III) complexes have been received more interest because of their strong red luminescence emission

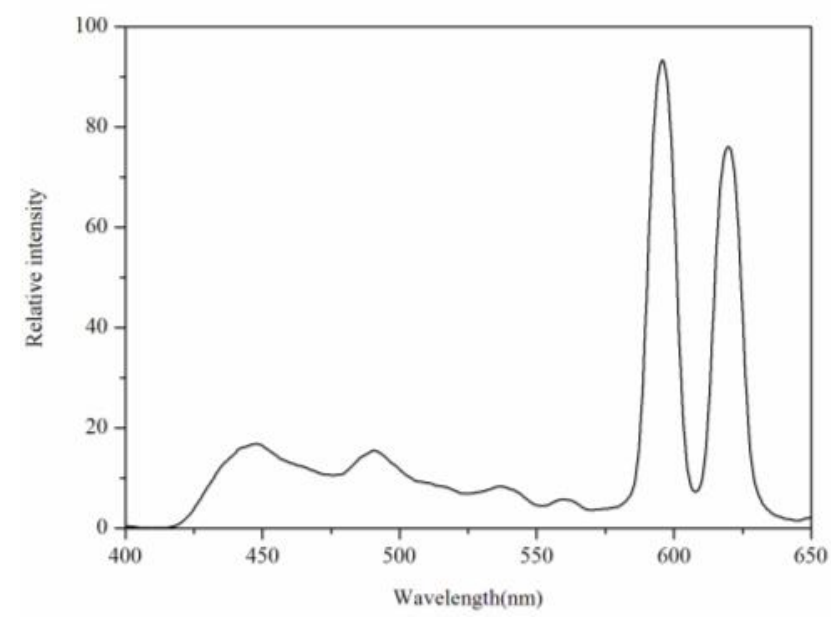

Figure 5. The emission spectrum of 1 . The excitation and emission slit widths were $5 \mathrm{~nm}$
[21]. Figure 5 displays the luminescence emission spectrum of complex $\mathbf{1}$ in solid state, it can be seen that the complex 1 gives two weak peaks (448 nm and $491 \mathrm{~nm}$ ) and two strong peaks $(596 \mathrm{~nm}$ and $620 \mathrm{~nm}$ ) from excitation at $279 \mathrm{~nm}$.

\section{Conclusions}

In summary, a new Eu(III) complex has been synthesized and its structure has been determined by elemental analysis, IR, luminescent and X-ray single crystal diffraction analysis. The catalytic activity for $\mathrm{A}^{3}$ coupling reaction using $\mathrm{Eu}(\mathrm{III})$ complex as catalyst is a higher yield of propargylamine than blank.

\section{Acknowledgement}

This project was supported by the Science Foundation of Weifang.

\section{References}

[1] Botelho, M.B.S., De Queiroz, T.B., Eckert, H., De Camargo, A.S.S. (2016). Efficient Luminescent Materials Based on the Incorporation of a $\mathrm{Eu}(\mathrm{III})$ Tris-(bipyridinecarboxylate) Complex in Mesoporous Hybrid Silicate Hosts. Journal of Luminescence, 170: 619-626.

[2] Chen, M.M., Li, H.X., Lang, J.P. (2016). Two Coordination Polymers and Their Silver(I) Doped Species: Synthesis, Characterization, and High Catalytic Activity for the Photodegradation of Various Organic Pollutants in Water. European Journal of Inorganic Chemistry, 2016: 2508-2515.

[3] Yuan, F., Zhang, L., Hua, H.M., Bai, C., Xue, G.L. (2017). Four New Coordination Polymers based on Carboxyphenyl- Substituted Dipyrazinylpyridine Ligand: Syntheses, Structures, magnetic and luminescence Properties. Journal of Molecular structure, 1128: 385-390.

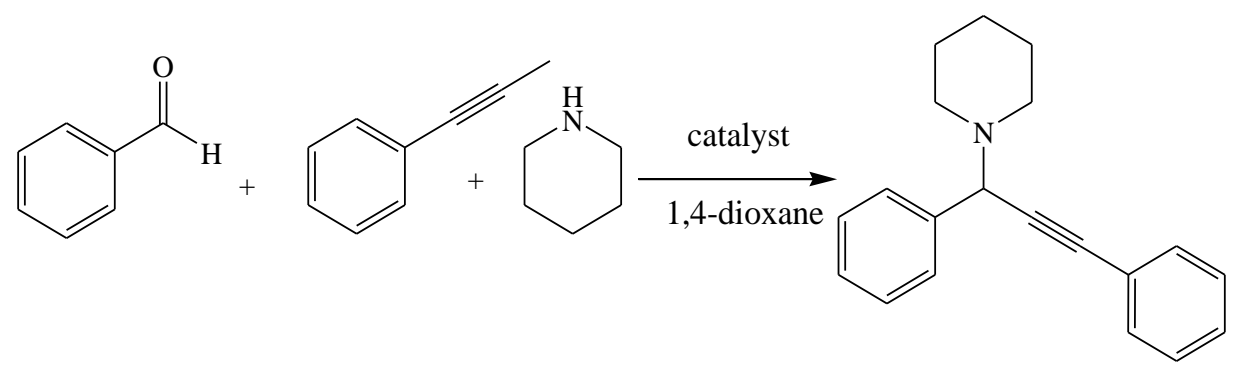

Figure 4. $\mathrm{A}^{3}$ coupling reaction of benzaldehyde, piperidine, and phenylacetylene using $\mathrm{Eu}(\mathrm{III})$ complex as catalyst 
[4] Ghandour, Y., Ben Khelifa, A., Belkhiria, M.S., Daiguebonne, C., Freslon, S., Guillou, O., Roisnel, T. (2016). Crystal Structure, Physico-chemical and Catalytic Properties of Two Organic-inorganic Hybrid Polyoxometallate-based Lanthanide Complexes. Polyhedron, 115: 1-8.

[5] Gusev, A.N., Hasegawa, M., Shul'gin, V.F., Nishchymenko, G., Linert, W. (2014). Photophysical studies on Ternary Mixed Ligand Europium Complexes Containing Pyridyltriazolylmethane and 1,3-Diketonate Ligands. Inorganica Chimica Acta, 414: 7177

[6] Lee, J.C., Jeong, Y.K., Kim, J.M., Kang, J.G. (2014). Sensitized Luminescence of $\mathrm{Eu}(\mathrm{III})$ Complexes with Schiff-base and 1,10Phenanthroline: Role of Schiff-base as a Sensitizer. Spectrochimica Acta, Part A: Molecular and Biomolecular Spectroscopy, 124: 256-264.

[7] Taha, M., Khan, I., Coutinho, J.A. (2016). Complexation and Molecular Modeling Studies of Europium (III)-gallic acid-amino Acid Complexes. Journal of Inorganic Biochemistry, 157: 25-33.

[8] Rao, D.R.M., Rawat, N., Sawant, R.M., Manna, D., Ghanty, T.K., Tomar, B.S. (2012). Thermodynamic Study of Eu(III) Complexation by Pyridine Monocarboxylates. The Journal of Chemical Thermodynamics, 55: 67-74.

[9] Tai, X.S., Wang, G.L., Liu, Y.Y. (2015). Synthesis and Crystal Structure of a $\mathrm{Na}(\mathrm{I})$ Complex with 4,4'-Bipyridine and 2-Formylbenzenesulfonate-hydrazine, Scientific Study \& Research: Chemistry \& Chemical Engineering, Biotechnology, Food Industry, 16: $173-177$.

[10] Da, S.S.L., Almeida, M.C., Lemos, T.L., Ribeiro, P.R., De Brito, E.S., Silva, V.L., Silva, A.M., Braz-Filho, R., Costa, J.G., Rodrigues, F.F., Barreto, F.S., De Moraes, M.O. (2016). Synthesis, Antibacterial and Cytotoxic Activities of New Biflorin-based Hydrazones and Oximes. Bioorganic and Medicinal Chemistry Letters, 26: 435-439.

[11] Hapuarachchige, S., Bryant, B.K., Arterburn, J.B. (2014). Synthesis of (Pyridin-2YL)hydrazone Rhenium(I) Tricarbonyl Complexes that Exhibit $\mathrm{pH}$-sensitive Fluorescence. Chemistry of Heterocyclic Compounds, 50: 254-263.
[12] Ghorbanloo, M., Bikas, M., Malecki, G. (2016). New Molybdenum(VI) Complexes with Thiazole-hydrazone Ligand: Preparation, Structural Characterization, and Catalytic Application in Olefin Epoxidation. Inorganica Chimica Acta, 445: 8-16.

[13] Liu, L.L., Tai, X.S., Zhang, N.N., Meng, Q.G., Xin, C.L. (2016). Supported Au/MIL-53(Al): A Reusable green solid Catalyst for the Threecomponent Coupling Reaction of Aldehyde, Alkyne, and Amine. Reaction Kinetics, Mechanisms, and Catalysis, 119: 335-348.

[14] Palchak, Z.L., Lussier D.J., Pierce C.J., Larsen C.H. (2015). Synthesis of Tetrasubstituted Propargylamines from Cyclohexanone by Solvent-free Copper(II) Catalysis. Green Chemistry, 17(3): 1802-1810.

[15] Tai, X.S., Liu, L.L., Yin, J. (2014). Synthesis, Crystal Structure of Tetra-Nuclear Macrocyclic $\mathrm{Cu}$ (II) Complex Material and Its Application as Catalysts for $\mathrm{A}^{3}$ Coupling Reaction. Journal of Inorganic and Organometallic Polymers and Materials, 24(6):1014-1020.

[16] Borah, B.J., Borah, S.J., Saikia, K., Dutta, D.K. (2014). Efficient One-pot Synthesis of Propargylamines Catalysed by Gold Nanocrystals Stabilized on Montmorillonite. Cata-lysis Science \& Technology, 4(11): 40014009.

[17] Berrichi, A., Bachir, R., Benabdallah, M., Choukchou-Braham, N. (2015). Supported Nano Gold Catalyzed Three-component Coupling Reactions of Amines, Dichloromethane and Terminal Alkynes (AHA). Tetrahedron Letters, 56: 1302-1306.

[18] Sheldrick, G.M. (1997). SHELXL-97, Program for Crystal Structure Solution. University of Göttingen: Göttingen, Germany.

[19] Sheldrick, G.M. (1997). SHELXTL-97, Program for Crystal Structure Refinement. University of Göttingen: Göttingen, Germany.

[20] Liu, L.L., Zhang, X., Gao, J.S., Xu, C.M. (2012). Engineering Metal-organic Frameworks Immobilize Gold Catalysts for Highly Efficient One-pot Synthesis of Propargylamines. Green Chemistry, 14: 17101720. 